\title{
Flow Analysis: Looking Back and Forward
}

\author{
Fábio R. P. Rocha* \\ Centro de Energia Nuclear na Agricultura, Universidade de São Paulo, \\ CP 96, 13400-970 Piracicaba-SP, Brazil
}

\begin{abstract}
Flow analysis changed significantly the way of performing chemical analysis by (i) mechanization of analytical procedures in a continuous flowing stream with minimal sample intercontamination; (ii) analytical measurements without attaining chemical equilibria, thus increasing the applicability of kinetic methods and allowing the exploitation of non-quantitative processes; (iii) exploitation of concentration gradients and (iv) use of unstable reagents or measurement of unstable products. Flow-based procedures are generally characterized by high sample throughput, improved precision, minimized waste generation, and often better selectivity. The performance of detection systems is usually improved because of the in-line sample conditioning and measurements under reproductive dynamic conditions and timing. However, after achieving several innovations, perspectives for further developments have been questioned mainly because of the recent decrease in the number of publications on flow analysis. The aim of this review is then to discuss the impact of flow-based methods on chemical analysis, emphasizing recent applications and developments, including miniaturization, bioanalysis, microextractions, green analytical chemistry and synergic hyphenation with other techniques and processes. The author's personal view about research perspectives in the field is also presented.
\end{abstract}

Keywords: flow injection analysis, sequential injection analysis, in-line microextraction, automation, chemical assays

\section{Introduction}

Flow analysis is a versatile approach for automation in analytical chemistry, which has undergone amazing development during the last decades. In this process, the sample is either continuously pumped or processed as a discrete aliquot inserted into a carrier stream. Processes such as chemical derivatizations, separations, analyte concentration/separations or controlled dilutions are efficiently carried out under highly reproductive mixing conditions and timing. As samples are handled in a closed system, risks to the analyst and of sample contamination are diminished. Typically, highly precise results are attained with relatively high sample throughputs, as well as minimized reagent consumption and waste generation. In short, flow analysis was defined as "the art of performing analytical chemistry in flowing streams" to perform chemistry". ${ }^{3}$

Developments in flow analysis have implied in breaking several paradigms and some aspects of analytical chemistry

*e-mail: frprocha@cena.usp.br

This paper is part of the PubliSBQ Special Issue "IUPAC-2017" (http://publi.sbq.org.br/) started to be seen in a different perspective. Historical aspects, ${ }^{4}$ fundamentals, and applications of flow analysis were properly discussed, ${ }^{1,3}$ including an online available tutorial. ${ }^{1}$

Brazilian researchers have effectively contributed to the inception and development of flow injection analysis (FIA), 5 the most successful approach involving unsegmented flows, and the main Brazilian contributions were critically discussed some years ago. ${ }^{6}$ The innovations encompassed novel flow approaches (e.g., merging zones, ${ }^{7}$ zone trapping, ${ }^{8}$ zone sampling, ${ }^{9}$ monosegmented flow analysis, ${ }^{10}$ multicommutation, ${ }^{11}$ and flow-batch analysis), ${ }^{12}$ as well as the pioneering coupling to different detection techniques (e.g., flame atomic absorption spectrometry (FAAS),${ }^{13}$ inductively coupled plasma optical emission spectrometry (ICP OES), ${ }^{14}$ turbidimetry ${ }^{15}$ and enthalpimetry $)^{16}$ and processes (e.g., ion-exchange ${ }^{17}$ and liquid-liquid extraction).$^{18}$ More recent advances include new alternatives for miniaturization (microFIA $^{19}$ and micro-flow batch analyzers), ${ }^{20}$ exploitation of detection by digital images ${ }^{21}$ as well as stepwise elution in sequential injection chromatography. ${ }^{22}$

This work is a critical overview of the main contributions of flow analysis to modern analytical chemistry, also 
highlighting the current status of this research field based on a literature survey. Recent innovations and the author's personal view about research perspectives in the field are also presented.

\section{Looking Backward: The Impact of Flow Analysis on Analytical Chemistry}

During first half of last century, chemical analyses were essentially carried out under equilibrium conditions, involving quantitative analyte conversions and exhaustive extractions. The analytical procedures were inherently timeconsuming and demanded intensive analyst interventions. Moreover, large amounts of residues were usually generated. With the inception of flow analysis in the fifties, automation became simpler and less expensive, and the kinetic aspects related to chemical analysis were gradually reinforced.

Before the proposal of flow analysis, mechanization of analytical procedures relied on discrete analyzers, in which each sample was processed in an independent vessel aiming at to avoid cross-contamination. This is an effective approach still widely used, mainly in laboratories devoted to large scale clinical analysis in which high sample throughput is needed. However, discrete analyzers present high mechanical complexity, which increases acquisition and maintenance costs. The first paradigm broken in the development of flow analysis was then the sample processing in the same compartment (typically a polymeric tube). The first approach, termed continuous flow analysis (and further segmented continuous flow analysis, SCFA), involved air segmentation to minimize sample broadening and thus cross contamination. ${ }^{23}$ Sample and reagents were continuously pumped, mixed, and segmented by air bubbles, which often were removed before measurement to avoid spurious signals. Long sample residence times (e.g., 10-15 min) were often required to carry out measurements under chemical and physical equilibria, which at that time was considered to be essential. Sampling rates within $30-50 \mathrm{~h}^{-1}$ were usually achieved by the simultaneous sample processing enabled by the negligible sample dispersion. The potential to achieve high sample throughput was improved with the advent of multichannel analyzers. ${ }^{24}$

The inception and earlier development of SCFA by Skeggs in the fifties was described in an interesting article..$^{25}$ At that time, Skeggs was responsible for a clinical analysis laboratory of a 1000-bed hospital, and only 3-4 analysts were accountable for all the steps involved in chemical analysis, including collecting blood samples. Hundreds of manual operations had to be performed daily, and erroneous results were frequent despite analysts' efforts.
Skeggs started then to "dream of a machine that would do analysis without error" and imagined that "analyses could be done in a continuously flowing stream rather than batchwise or discreetly". From this idea, he built up the first SCFA prototype, which was exploited for the colorimetric determination of urea, involving in-line dialysis, to proof the concept. ${ }^{23}$ Further, the equipment was commercialized as AutoAnalyzer ${ }^{\circledR}$ and widely used mainly for clinical and environmental analysis.

About 20 years after the inception of SCFA and following the proposals of some approaches involving unsegmented flows, ${ }^{26,27}$ the term flow injection analysis (FIA) was coined by Ruzicka and Hansen. ${ }^{28}$ They also established the fundamentals of FIA and jointly with other co-workers demonstrated several of its potentialities to large scale chemical analysis. Essentially, FIA involves sample handling without air segmentation, thus broken the paradigm established by SCFA. A discrete sample aliquot (typically from 10 to $500 \mu \mathrm{L}$ ) is inserted into a continuously flowing carrier stream. Without air segmentation, sample dispersion occurs at the interfaces, but in a controlled and reproducible way. This generates a transient signal recorded as a peak, whose height reflects the analyte concentration in the sample. Measurements can be carried out in any portion of the monitored dispersed sample and this paved the way for exploitation of the reproducible concentration gradients. The applicability of this approach was demonstrated for e.g., controlled dilutions, calibration (or re-calibration) with a single reference solution, implementation of the standard additions method, selectivity evaluation, and titrations. ${ }^{29}$ The latter application can be exemplified by a recently proposed approach involving tracer-monitored flow titrations. ${ }^{30}$ Concentrations of an indicator and a tracer (added to the sample or the titrant) are continuously monitored (e.g., by multi-channel spectrophotometry), when a sample aliquot is inserted into a titrant stream. Sample and titrant undergo dispersion at the interfaces and because of the mutual mixing, equivalence conditions are attained at both the front and the tail of the sample zone. A mixing chamber was exploited to minimize the effects of differences in diffusion of the titrant, analyte, and tracer, a potential source of systematic errors. The concentration profile of the tracer was considered to estimate the instant sample and titrant volumetric fractions without the need for volume, mass or peak width measurements. Thus, the analyte concentration in the sample may be estimated from a simple sample injection, without the need for any calibration step, thus being a true titration according to IUPAC definition.

Other interesting aspect reinforced by the advent of FIA was the exploitation of kinetic aspects. This brought a new dimension to analytical chemistry and made feasible 
several processes considered inviable when measurements were taken under equilibrium conditions. Without air segmentation, the residence times had to be significantly lower (typically 10-30 s) in order to avoid excessive sample dispersion. Thus, measurements are often carried out without attaining chemical equilibrium, which is feasible because of the highly reproducible timing, a characteristic potentialized by the advent of flow programming. ${ }^{31}$ In this sense, the most frequent approaches were fixed-time ${ }^{1-3}$ and stopped-flow methods. ${ }^{32}$ The former is the most usual approach and typically yields a reproducible response characteristic of a defined sample residence time, whereas the later allows the continuous monitoring of a product during its formation or degradation. FIA is then inherently a kinetic analytical approach and, in this context, applications encompass kinetic discrimination either to improve selectivity ${ }^{33}$ or aiming at simultaneous determinations. ${ }^{34,35}$ This exploits measurement of a reaction product before formation of an analogous with the interfering species or by exploiting differences in the rate of formation of these products. Determination of kinetic parameters (e.g., rate constants $)^{36}$ is also feasible. Moreover, flow analysis is a very suitable tool for exploitation of catalytic methods, ${ }^{37}$ which are typically characterized by high sensitivity.

Exploitation of unstable reagents and measurements of unstable reaction products is also relevant. ${ }^{38}$ In this sense, strong oxidants (e.g. $\mathrm{Ag}^{\mathrm{II}}, \mathrm{Mn}^{\mathrm{III}}, \mathrm{Co}^{\mathrm{III}}$, and $\mathrm{Br}_{2}$ ) or reductants (e.g., $\mathrm{Cr}^{\mathrm{II}}, \mathrm{U}^{\mathrm{III}}, \mathrm{Mo}^{\mathrm{III}}$ ), as well as radicals (e.g., $\mathrm{OH}^{\bullet}, \mathrm{SO}_{4}^{-\bullet}$, ${ }^{\bullet} \mathrm{CH}_{2} \mathrm{OH}$, and ${ }^{\bullet} \mathrm{COOH}$ ) may be reproductively generated in flow systems by chemical, ${ }^{38}$ electrochemical ${ }^{38}$ or photolytic processes. ${ }^{39}$ Thus, the need for frequent standardization, difficulties with storage and time consumption in reagent preparation is avoided. This achievement paved the way to revisit a diversity of chemical reactions as well as the analytical exploitation of novel processes. Moreover, reaction intermediates ${ }^{40}$ or suspensions ${ }^{15}$ can be reproductively measured with good precision before their degradation or precipitate settlement. In relation to formation of slightly soluble species, crystal growth under highly reproducible conditions is also favorable. In this context, because of the inherent reproducible timing and the improved mixing conditions, analytical applications of chemiluminescence ${ }^{40}$ were boosted by the development of FIA (Figure 1).

Because of the precise time control, flow analysis makes feasible the analytical exploitation of non-quantitative processes, such as incomplete chemical derivatizations or partial analyte extractions, as demonstrated in several applications in the literature. For example, an incomplete photochemical conversion was exploited for phosphorus fractionation in cereals. ${ }^{41}$ In these samples, organic

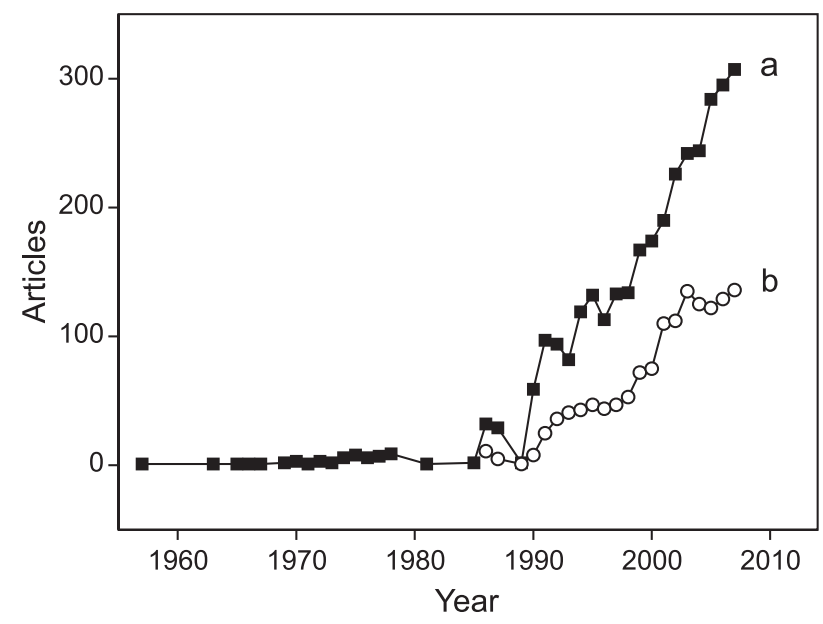

Figure 1. Number of scientific articles on analytical applications of chemiluminescence (a) and its application as detection in flow analysis (b). Source: ISI Web of Science database.

phosphorus is essentially found as phytic acid, whose mineralization by usual approaches (e.g., microwaveassisted acid digestions) is hard to achieve. On the other hand, this species is fast converted to phosphate in the reaction with sulfate and hydroxyl radicals. These strong oxidants were photochemically produced in a multipumping flow system ${ }^{42}$ from a persulfate solution (equations 1 and 2) and fractionation was accomplished by measuring the phosphate amount by the molybdenum blue method with and without adding persulfate to the sample zone. Good precision (coefficient of variation $(\mathrm{CV})$ within 1.2 and $3.6 \%$ ) and reliable results were achieved even with a conversion rate of phytic acid to phosphate estimated at $48 \%$. The approach also minimized significantly the reagent consumption (e.g., $790 \mu \mathrm{K} \mathrm{K}_{2} \mathrm{~S}_{2} \mathrm{O}_{8}$ per determination) and waste generation ( $2.5 \mathrm{~mL}$ per determination).

$\mathrm{S}_{2} \mathrm{O}_{8}^{2-}+\mathrm{hv} \rightarrow 2 \mathrm{SO}_{4}^{-\bullet}$
$\mathrm{SO}_{4}^{-\bullet}+\mathrm{H}_{2} \mathrm{O} \rightarrow \mathrm{HSO}_{4}^{-}+\mathrm{OH}^{\bullet}$

Microextractions have replaced exhaustive extractions in analytical chemistry because of several advantages such as low solvent (or sorbent) consumption and high sample throughput. Microextractions have been also usual in flow analysis because of the capability to carry out partial yet reproducible analyte extractions. For example, this process was exploited for the determination of the iodine value in biodiesel, ${ }^{43}$ which indicates the unsaturation degree and thus the oxidative stability of the biofuel. With a multicommuted flow system, an aqueous triiodide solution was intercalated between two biodiesel aliquots. Iodine was then partially extracted to the organic phase (equation 3), where the halogenation reaction of the alkyl esters took place (equation 4). Discoloration of the 
aqueous reagent was proportional to the iodine value of the biodiesel. With this strategy, results for biodiesel produced from different feedstocks agreed with those obtained by the reference method, which involved titration in organic medium and consumed large solvent amounts. Further experiments demonstrated that matrix effects, which were negligible in the flow-based procedure, were relevant when measurements were carried out batchwise under equilibrium conditions.

$\mathrm{I}_{3(\mathrm{aq})}^{-} \rightleftharpoons \mathrm{I}_{(\mathrm{aq})}^{-}+\mathrm{I}_{2(\mathrm{aq})} \rightleftharpoons \mathrm{I}_{2(\mathrm{org})}$

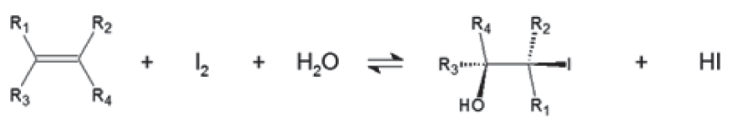

By considering the previous discussion, one can conclude that the impact of flow analysis in analytical chemistry has been really impressive. This is reflected in more than 23000 publications in the field from authors worldwide, ${ }^{1}$ at least 20 textbooks, two regular international conference series (Flow Analysis and International Conference on Flow Injection Analysis), a lot of academic thesis, reference analytical methods, such as those reported in the Standard Methods for the Examination of Water and Wastewater, ${ }^{44}$ commercially available equipment, and a specific international journal (Journal of Flow Injection Analysis, ${ }^{45}$ published by The Japanese Association for Flow Injection Analysis).

\section{Looking Forward: Research Perspectives on Flow Analysis}

To talk about future is ever a hard task and as stressed by John Sculley (former CEO of Apple and Pepsi): "tell what the future is gonna be is the easy part. The hard part is telling when its gonna happen...". Moreover, discussions about the future of flow analysis are not novel. In fact, this has been the theme of some lectures and review articles published by experts in the field (e.g., Snyder, ${ }^{46}$ Hansen, ${ }^{47}$ and Hansen and Miró), ${ }^{33}$ including a recent publication by J. Ruzicka entitled "From continuous flow analysis to programmable Flow Injection Techniques. A history and tutorial of emerging methodologies". ${ }^{31}$ A critical view of flow analysis was also recently presented in an editorial in this journal. ${ }^{48}$

This discussion has been motivated by the decrease in the number of publications and probably also in the number of researchers acting in the field of flow analysis. Whereas the overall scientific production in analytical chemistry and in some well-established subareas (e.g., electroanalysis and chromatography) is significantly increasing and the number of articles in other subareas (e.g., atomic spectrometry) is nearly constant, the scientific production in flow analysis has been deeply decreased since 2008 (Figure 2). This indicates that the main aspects inherent to flow analysis were already demonstrated, including applications to diverse analytical problems. By taking this scenario into account, one may ask about the perspective of researches in the field.

The panorama of the scientific production in flow analysis would be probably more favorable if the works related to microfluidic devices were taken into account. Indeed, scientific production in this field has increasing significantly-a literature survey in ISI Web of science database restricted to the contributions in journals classified as "chemical, analytical" returned 8118 papers in the last 20 years (e.g., 150, 380, and 722 papers per year in 2002, 2006, and 2016, respectively). However, even articles related to flow approaches (or involving the same principles, e.g., controlled dispersion/diffusion, reproducible timing, and sample injection) did not mention terms related to flow analysis either in the title or in the keywords. ${ }^{49-51}$ In fact, this explicit correlation was observed in only $4.6 \%$ of the articles. This indicates that flow analysis is presently considered a well-established approach and emphasis has been given to the design of microfluidic devices as well as novel applications. A similar comment could be done in relation to flow chemistry, which essentially exploits the same concepts involved in flow analysis, although the previous developments in the analytical field have been rarely mentioned. ${ }^{52} \mathrm{~A}$ recent work entitled "Flow chemistry vs. flow analysis" ${ }^{53}$ highlighted the similarities observed in the flow approaches designed either to analytical or synthetic aims as well as the synergistic developments which could be achieved by considering the advances in these fields in conjunct. The author also envisioned that the term "flow chemistry" will encompass the approaches for analytical and synthetic purposes in the future.

As shown in Figure 3, the number of citations in flow analysis has been constant, nevertheless relatively high in the last five years. Of course, with the increased number of articles, a constant number of citations implies a decrease in the mean citation rate. However, overall the mean citation per article in this area is about 24, which is higher than the mean citation of a general article in analytical chemistry. This indicates that the articles on flow analysis still cause a significant scientific impact.

Nowadays, it is more difficult to achieve an innovative research in flow analysis, but the situation is not different in other well-established subareas of analytical chemistry. Discussions about the continuity of the research are not so frequent in other subareas (such as chromatography, electroanalysis and atomic spectrometry) perhaps because 

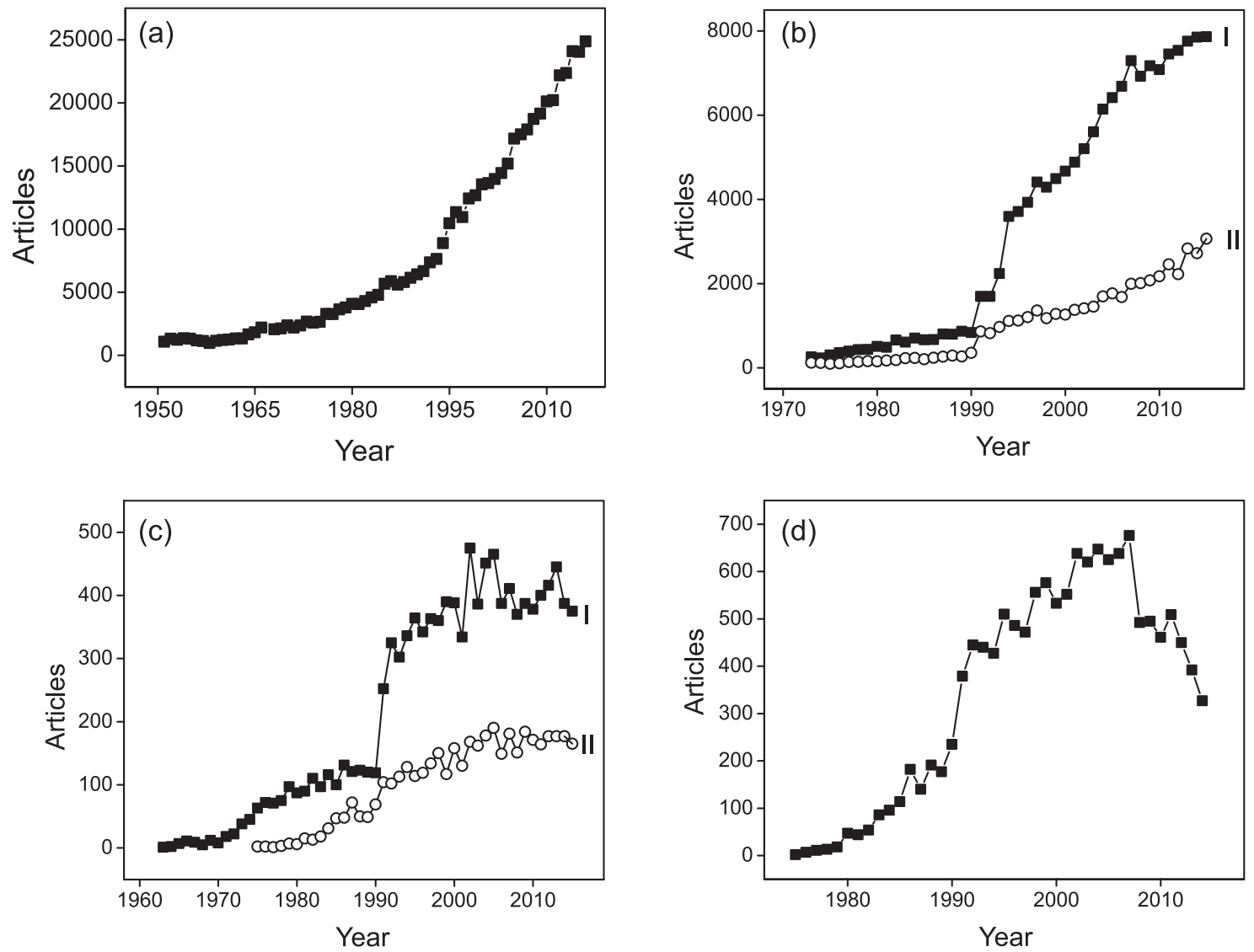

Figure 2. Number of scientific articles on (a) analytical chemistry; (b) chromatography (I) and electroanalysis (II); (c) atomic spectrometry-(I) atomic absorption and (II) inductively coupled plasma optical emission; (d) flow analysis. Source: ISI Web of Science database.

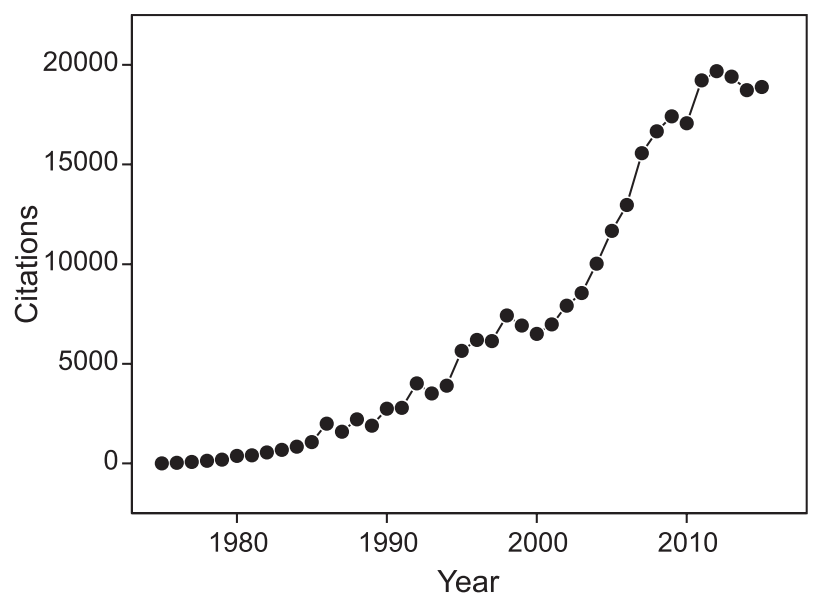

Figure 3. Evolution of the citations on flow analysis. Source: ISI Web of Science database.

these techniques are more usual in routine analysis and thus there are competitive markets for equipment. These techniques and market have been constantly revitalized by technological innovations such as continuous source atomic absorption spectrometers and tandem mass spectrometers as powerful detectors in chromatography and ICPs. In relation to flow analysis, proposal of novel flow modalities and processes has been typically restricted to research labs and often encompasses a few research groups in the field.

As discussed by several authors, the development of unsegmented flow analysis comprises three generations: flow injection analysis, sequential injection analysis, and new approaches such as bead injection and lab-onvalve. In another perspective, we can consider other classification, with a partial overlap between the groups. The initial focus was on the improvement of analytical performance (mainly sampling rate and precision) of classical analytical methods. ${ }^{15,18,54}$ The $2^{\text {nd }}$ trend was exploitation of unconventional processes, such as incomplete derivatizations ${ }^{8,32}$ and partial extractions (e.g., dialysis ${ }^{55}$ or gas-diffusion), ${ }^{56}$ whereas the $3^{\text {rd }}$ focus was on proposal of new flow modalities, such as sequential injection analysis (SIA), ${ }^{57}$ multicommutation, ${ }^{11}$ multipumping, ${ }^{42}$ flow-batch analysis, ${ }^{12}$ multisyringe flow injection analysis, ${ }^{58}$ with some advantages and similarities. The $4^{\text {th }}$ group will probably involve the same trends highlighted in modern analytical chemistry, with emphasis on miniaturization, green chemistry, novel strategies for microextractions, chemical speciation, bioanalysis, and analytical solutions devoted to complex samples (or complex analytical 
problems). As noted in recent years, ${ }^{59-61}$ hyphenation of flow systems to separation techniques aiming at on-line sample pretreatment probably will remain a fertile research field. Some recent examples will be discussed next.

Miniaturization brings several advantages to flow analysis, such as portability and minimization of sample and reagent consumptions as well as waste generation. ${ }^{19}$ Moreover, novel approaches are feasible because of the typical dispersion pattern and the large surface-to-volume ratios inherent to microchannels. In this sense, several approaches have been exploited to build up micro-flow analyzers. ${ }^{19,20,62,63}$ A recent proposal refers to chip-based manifolds obtained with $3 \mathrm{D}$ printers, which can also integrate the flow cell. An example of application is the catalytic fluorimetric determination of iodide in seawater, achieving a detection limit of $300 \mathrm{ng} \mathrm{L}^{-1} \cdot{ }^{64}$ Other applications discussed in the present review also exploited miniaturized systems. ${ }^{51,61,65}$

Flow analysis has been pointed out as a powerful tool for green analytical chemistry ${ }^{66}$ Minimization of reagent consumption and waste generation is inherent to the process, but it is more evident in micro-flow analyzers, ${ }^{19,51}$ as well as in some approaches such as merging zones, ${ }^{7}$ SIA,${ }^{57}$ multicommutation, ${ }^{11}$ and multipumping. ${ }^{42}$ Moreover, ingenious approaches such as exploitation of hanging drops ${ }^{67}$ reuse of chemicals (e.g., an immobilized reagent used for reversible analyte sorption) ${ }^{35}$ and even reagentless procedures are feasible. The later can be illustrated by the eco-friendly determination of free glycerol in biodiesel based on water-cavitation sonoluminescence. ${ }^{68}$ Water was used for analyte extraction and its sonolysis generates excited hydroxyl radicals, whose luminescence was quenched by the analyte. All the process was carried out in a flow-batch chamber, which included a piezoelectric device and an optical fiber adapted to a lab-made luminometer. With this reagentless approach, as low as $60 \mathrm{pg}$ of glycerol can be measured by using only $15 \mu \mathrm{L}$ of sample.

Relatively to solid-phase microextractions, the ability of flow analyzers for the highly reproductive handling of suspensions has been exploited in novel approaches involving movable sorbents, such as beads ${ }^{69}$ and fluidized beds. ${ }^{70}$ This circumvents drawbacks related to excessive backpressure and preferential pathways, also improving the analyte-sorbent interaction. Applications of solid-phase microextractions in flow systems encompass miniaturization, ${ }^{71,72}$ bioanalysis,${ }^{72}$ and chemical speciation. ${ }^{73}$ Emphasis has been given to selective materials, biosorbents, and nanomaterials, including modified magnetic nanoparticles..$^{71,74}$ These materials are dispersed into the sample and further separated by means of an external magnet, thus favoring the analyte sorption and avoiding the increase in backpressure. On the other hand, monolithic columns and fused-core particles have been exploited for low-pressure chromatographic separations, especially in sequential injection systems (named as sequential injection chromatography). ${ }^{75}$ Potentialities and applications on this subject have been revised ${ }^{59}$ and recent innovations include on-line sample preparation before chromatographic separation ${ }^{76}$ and on-column preconcentration of the analytes. ${ }^{77}$

Novel strategies have been also proposed for liquid-liquid microextractions, including mechanization of dispersive liquid-liquid microextraction ${ }^{78,79}$ and cloud point extraction. ${ }^{80}$ In the versatile lab-in-syringe modality, ${ }^{78,80}$ all involved processes (e.g., chemical derivatization, solvent dispersion, induction of the cloud point, phase separation, and detection) can be carried out inside a syringe. On the other hand, exploitation of pulsed flows (inherent to the multipumping approach) was beneficial for reproductive dispersion of the extractor in dispersive liquid-liquid microextraction. ${ }^{79}$ Liquid-liquid microextraction has also been carried out in flow-based microfluidic devices, thus reducing the solvent consumption to a few nanoliters. ${ }^{51}$

Non-chromatographic procedures have been developed for chemical speciation. In a recent application, in-line UV photooxidation (under heterogeneous catalysis by $\mathrm{TiO}_{2}$ ), selective hydride generation, and arsine trapping into oxidized carbon nanotubes were exploited for arsenic speciation by graphite furnace atomic absorption spectrometry (GFAAS) ${ }^{81}$ Speciation was accomplished in a miniaturized flow system by sample processing with and without photooxidation and before and after reduction of $\mathrm{As}^{\mathrm{V}}$ to $\mathrm{As} \mathrm{s}^{\mathrm{II}}$. A detection limit as low as $0.78 \mathrm{ng} \mathrm{L}^{-1}$ was achieved by consuming only a few microliters of each reagent.

An immunoprecipitation assay for diabetes diagnosis can exemplify a recent bioanalytical application of flow analysis. ${ }^{82}$ It was based on the nephelometric detection of a suspension yielded by the interaction of human albumin with its antibody carried out in a multicommuted flow system. This illustrates the exploitation of a well-known process (i.e., the reproductive management of suspensions under flow conditions) for an actual and relevant application. Another example involves an amperometric immunoassay for determination of residues of an organochloride pesticide (2,6-dichlorobenzamide) carried out in a microfluidic device. ${ }^{65}$ The immunosorbent was covalently immobilized in one of the chip channels and the long-term stability required for at line monitoring of the pollutant in water supplies was achieved.

Flow systems have also been useful for sample preparation, either involving sample mineralization or clean-up as well as analyte extraction/derivatization. In fact, hyphenation of flow 
analysis to separation techniques has resulted in a synergistic improvement of analytical performance and the number of applications has increasing recently. In this context, the potentiality, interfacing, and applications of FIA/SIA for e.g., on-line membrane-assisted sample treatment by dialysis or gas-diffusion, preconcentration by solid-phase extraction (SPE), and analyte derivatization in capillary electrophoresis were reviewed. ${ }^{83}$ Flow analysis has been hyphenated to both gas and liquid chromatography. A recent example of the former is exploitation of a multisyringe flow system for sample treatment before determination of the beta-blockers atenolol and propranolol in human plasma. ${ }^{60}$ The in-line sample treatment involved deproteinization by SPE with a restricted access material and chemical derivatization under microwave-assisted heating to yield trimethylsilated derivatives. A sampling rate of $7 \mathrm{~h}^{-1}$ was achieved by performing sample treatment and separation by gas chromatography simultaneously. In relation to high performance liquid chromatography, an illustrative example is the coupling of a (3D printed) micro flow injection system for clean-up of saliva and urine samples and preconcentration of emerging pollutants (triclosan and methy-, ethyl-, propyl-, phenyl-, and butylparaben) before chromatographic separation. ${ }^{61}$ Sorption of the analytes was carried out in magnetite nanoparticles modified with polyaniline packed into a SPE micro-column, thus achieving enrichment factors in the range 16-25. Sample treatment demanded $20 \mathrm{~min}$, but it was synchronized with the chromatographic separation aiming at better sample throughput.

These few recent examples illustrate the diversity of applications successfully carried out by exploiting the peculiar characteristics of flow analysis. A recent comprehensive review ${ }^{52}$ discussed other relevant applications and presented a list of 136 review articles focusing on different aspects and applications of flow analysis. Moreover, flow-based procedures have been widely used as analytical tools in several fields (e.g., environmental ${ }^{84}$ and food analysis), ${ }^{85}$ even without any reference in the title or in the keywords. This clearly states flow analysis as a well-established analytical tool. Because of the ability for effective management of samples and reagents under highly reproductive conditions, flow systems will probably remain as a unique tool to solve analytical problems.

\section{Conclusions and Perspectives}

The impact of flow analysis on the way chemical analysis is currently performed is not arguable, mainly in relation to exploitation on non-quantitative processes and unusual chemical reactions. These approaches are advantageous in relation to selectivity, precision, sample throughput, as well as minimization of reagent consumption and waste generation. On the other hand, numerical indicators have demonstrated the decrease of the number of published articles in recent years and one should not expect that this panorama will be significantly modified, unless other research groups start to act in the field. This can be achieved by carrying out researches at the interface, as has been demonstrated by bioanalytical applications and exploitation of flow systems for on-line sample pretreatment and analyte derivatization before chromatographic separation, mass spectrometric detection, or both.

An additional issue is if a large number of publications is really required. By considering the recent applications and trends, one may expect that the massive scientific production in flow analysis in the last three decades will be replaced by high quality innovative contributions, especially focusing on miniaturization, green analytical chemistry, microextractions, chemical speciation, bioanalysis, and sample preparation, or at the interfaces of these areas, as demonstrated in some examples previously discussed. In the author personal view, innovative contributions will remain attracting the interest of editors and readers of the main journals in analytical chemistry and this is more important than a large number of publications. New paradigms will have to be broken for innovative contributions and further developments in flow analysis. This will require creativity, knowledge, and focus on unsolved analytical problems. In this sense, flow analysis should be considered as one of the useful available tools to perform analytical chemistry.

\section{Acknowledgments}

The author acknowledges the organizers of the $46^{\text {th }}$ World Chemistry Congress and specially those of the Analytical \& Food Chemistry Symposia, Joaquim A. Nóbrega and Celio Pasquini for the invitation to the lecture. Elias A. G. Zagatto and Wanessa M. Mattos are thanked for critical comments. Fellowships and financial support from the Brazilian agencies CAPES, CNPq, and FAPESP are also acknowledged.

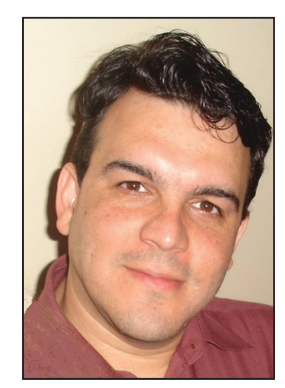

Fábio R. P. Rocha is graduated and PhD in Chemistry with professional experience in Analytical Chemistry. He is professor at the Center for Nuclear Energy in Agriculture/University of São PauloBrazil and his research is focused on analytical automation and 
instrumentation, flow analysis, optical methods of analysis, sample treatment, and green analytical chemistry. He has developed environmental friendly analytical procedures for diverse applications, including environmental, food, pharmaceutical, and fuel analysis.

\section{References}

1. http://www.flowinjectiontutorial.com, accessed on September $1,2017$.

2. Zagatto, E. A. G.; Oliveira, C. C.; Townshend, A.; Worsfold, P. J.; Flow Analysis with Spectrophotometric and Luminometric Detection, $1^{\text {st }}$ ed.; Elsevier: Amsterdam, 2012.

3. Karlberg, B.; Pacey, G. E.; Flow Injection Analysis: A Practical Guide; Elsevier: Amsterdam, 1989.

4. Stewart, K. K.; Talanta 1981, 28, 789.

5. Hansen, E. H.; Ruzicka, J.; TrAC, Trends Anal. Chem. 1983, 2, V.

6. Batista, A. D.; Sasaki, M. K.; Rocha, F. R. P.; Zagatto, E. A. G.; Analyst 2014, 139, 3666.

7. Bergamin-Filho, H.; Zagatto, E. A. G.; Krug, F. J.; Reis, B. F.; Anal. Chim. Acta 1978, 101, 17.

8. Krug, F. J.; Reis, B. F.; Giné, M. F.; Zagatto, E. A. G.; Ferreira, J. R.; Jacintho, A. O.; Anal. Chim. Acta 1983, 151, 39.

9. Reis, B. F.; Jacintho, A. O.; Mortatti, J.; Krug, F. J.; Zagatto, E. A. G.; Bergamin-Filho, H.; Pessenda, L. C. R.; Anal. Chim. Acta 1981, 123, 221.

10. Pasquini, C.; Oliveira, W. A.; Anal. Chem. 1985, 57, 2575.

11. Reis, B. F.; Giné, M. F.; Zagatto, E. A. G.; Lima, J. L. F. C.; Lapa, R. A. S.; Anal. Chim. Acta 1994, 293, 129.

12. Diniz, P. H. G. D.; Almeida, L. F.; Harding, D. P.; Araújo, M. C. U.; TrAC, Trends Anal. Chem. 2012, 35, 39.

13. Zagatto, E. A. G.; Krug, F. J.; Bergamin-Filho, H.; Jorgensen, S. S.; Reis, B. F.; Anal. Chim. Acta 1979, 104, 279.

14. Jacintho, A. O.; Zagatto, E. A. G.; Bergamim-Filho, H.; Krug, F. J.; Reis, B. F.; Bruns, R. E.; Kowalski, B. R.; Anal. Chim. Acta 1981, 130, 243.

15. Krug, F. J.; Bergamim-Filho, H.; Zagatto, E. A. G.; Jorgensen, S. S.; Analyst 1977, 102, 503.

16. Pasquini, C.; Oliveira, W. A.; Anal. Chim. Acta 1984, 156, 307.

17. Bergamin-Filho, H.; Reis, B. F.; Jacintho, A. O.; Zagatto, E. A. G.; Anal. Chim. Acta 1980, 117, 81.

18. Bergamin-Filho, H.; Medeiros, J. X.; Reis, B. F.; Zagatto, E. A. G.; Anal. Chim. Acta 1978, 101, 9.

19. Fonseca, A.; Raimundo, I. M.; Rohwedder, J. J. R.; Ferreira, L. O. S.; Anal. Chim. Acta 2007, 603, 159.

20. Monte, S. S.; Lima, M. B.; Andrade, S. I. E.; Harding, D. P.; Fagundes, Y. N. M.; Santos, S. R. B.; Lemos, S. G.; Araujo, M. C. U.; Talanta 2011, 86, 208.

21. Lima, M. B.; Andrade, S. I. E.; Barreto, I. S.; Almeida, L. F.; Araujo, M. C. U.; Microchem. J. 2013, 106, 238.
22. Rigobello-Masini, M.; Penteado, J. C. P.; Liria, C. W.; Miranda, M. T. M.; Masini, J. C.; Anal. Chim. Acta 2008, 628, 123.

23. Skeggs Jr., L. T.; Am. J. Clin. Pathol. 1957, 28, 311.

24. Thiers, R. E.; Bryan, J.; Oglesby, K.; Clin. Chem. 1966, 12, 120.

25. Skeggs Jr., L. T.; Clin. Chem. 2000, 46, 1425.

26. Pungor, E.; Fehér, Z. S.; Nagy, G.; Anal. Chim. Acta 1970, 51, 417.

27. White, V. R.; Fitzgerald, J. M.; Anal. Chem. 1972, 44, 1267.

28. Ruzicka, J.; Hansen, E. H.; Anal. Chim. Acta 1975, 78, 145.

29. Ruzicka, J.; Hansen, E. H.; Anal. Chim. Acta 1983, 145, 1.

30. Sasaki, M. K.; Rocha, D. L.; Rocha, F. R. P.; Zagatto, E. A. G.; Anal. Chim. Acta 2016, 902, 123.

31. Ruzicka, J.; Talanta 2016, 158, 299.

32. Ruzicka, J.; Hansen, E. H.; Anal. Chim. Acta 1979, 106, 207.

33. Hansen, E. H.; Miró, M.; TrAC, Trends Anal. Chem. 2007, $26,18$.

34. Fortes, P. R.; Meneses, S. R. P.; Zagatto, E. A. G.; Anal. Chim. Acta 2006, 572, 316.

35. Teixeira, L. S. G.; Rocha, F. R. P.; Korn, M.; Reis, B. F.; Ferreira, S. L. C.; Costa, A. C. S.; Talanta 2000, 51, 1027.

36. Echols, R. T.; Tyson, J. F.; Talanta 1994, 41, 1775.

37. Cerdà, V.; González, A.; Danchana, K.; Talanta 2017, 167, 733.

38. den Boef, G.; Anal. Chim. Acta 1989, 216, 289.

39. Rocha, D. L.; Kamogawa, M. Y.; Rocha, F. R. P.; Anal. Chim. Acta 2015, 896, 11.

40. Fletcher, P.; Andrew, K. N.; Calokerinos, A. C.; Forbes, S.; Worsfold, P. J.; Luminescence 2001, 16, 1.

41. Rocha, D. L.; Rocha, F. R. P.; Microchem. J. 2013, 109, 139.

42. Lapa, R. A. S.; Lima, J. L. F. C.; Reis, B. F.; Santos, J. L. M.; Zagatto, E. A. G.; Anal. Chim. Acta 2002, 466, 125.

43. Pereira, A. C.; Rocha, F. R. P.; Anal. Chim. Acta 2014, 829, 28.

44. Eaton, A. D.; Clesceri, L. S.; Greenberg, A. E.; Standard Methods for the Examination of Water and Wastewater, $19^{\text {th }} \mathrm{ed}$.; American Public Health Association: Washington, 1995.

45. http://jafia.kyushu-u.ac.jp/english/, accessed on December 10, 2017.

46. Snyder, L. R.; Anal. Chim. Acta 1980, 114, 3.

47. Hansen, E. H.; Talanta 2004, 64, 1076.

48. Zagatto, E. A. G.; J. Braz. Chem. Soc. 2015, 26, 831.

49. Jayawardane, B. M.; Wei, S.; McKelvie, I. D.; Kolev, S. D.; Anal. Chem. 2014, 86, 7274.

50. Beaton, A. D.; Siebena, V. J.; Floquet, C. F.A.; Waugh, E. M.; Bey, S. A. K.; Ogilvie, I. R.G.; Mowlem, M. C.; Morgana, H.; Sens. Actuators, B. 2011, 156, 1009.

51. Smirnova, A.; Mawatari, K.; Hibara, A.; Proskurnin, M. A.; Kitamori, T.; Anal. Chim. Acta 2006, 558, 69.

52. Trojanowicz, M.; Kolacinska, K.; Analyst 2016, 141, 2085.

53. Trojanowicz, M.; Talanta 2016, 146, 621.

54. Ruzicka, J.; Stewart, J. W. B.; Anal. Chim. Acta 1975, 79, 79.

55. Ruzicka, J.; Hansen, E. H.; Anal. Chim. Acta 1976, 87, 353. 
56. van der Linden, W. E.; Anal. Chim. Acta 1983, 151, 359.

57. Ruzicka, J.; Marshall, G. D.; Anal. Chim. Acta 1990, 237, 329.

58. Albertus, F.; Horstkotte, B.; Cladera, A.; Cerda, V.; Analyst 1999, 124, 1373.

59. Chocholous, P.; Solich, P.; Satínský, D.; Anal. Chim. Acta 2007, 600, 129.

60. Brunetto, M. R.; Clavijo, S.; Delgado, Y.; Orozco, W.; Gallignani, M.; Ayala, C.; Cerdà, V.; Talanta 2015, 132, 15.

61. Wang, H.; Cocovi-Solberg, D. J.; Hu, B.; Miró, M.; Anal. Chem. 2017, 89, 12541.

62. Cerdà, V.; Avivar, J.; Moreno, D.; Talanta 2017, 166, 412.

63. Miró, M.; Hansen, E. H.; Anal. Chim. Acta 2012, 750, 3.

64. Frizzarin, R. M.; Aguado, E.; Portugal, L. A.; Moreno, D.; Estela, J. M.; Rocha, F. R. P.; Cerdà, V.; Talanta 2015, 144, 1155.

65. Uthuppu, B.; Heiskanen, A.; Kofoed, D.; Aamand, J.; Jorgensen, C.; Dufva, M.; Jakobsen, M. H.; Analyst 2015, 140, 1616.

66. Melchert, W. R.; Reis, B. F.; Rocha, F. R. P.; Anal. Chim. Acta 2012, 714, 8 .

67. Liu, H. H.; Dasgupta, P. K.; TrAC, Trends Anal. Chem. 1996, $15,468$.

68. Diniz, P. H. G. D.; Pistonesi, M. F.; Araújo, M. C. U.; FernándezBand, B. S.; Talanta 2013, 114, 38.

69. Oliveira, H. M.; Miró, M.; Segundo, M. A.; Lima, J. L. F. C.; Talanta 2011, 84, 846.

70. Ribeiro, M. F. T.; Dias, A. C. B.; Santos, J. L. M.; Lima, J. L. F. C.; Zagatto, E. A. G.; Anal. Bioanal. Chem. 2006, 384, 1019.

71. Vidigal, S. S. M. P.; Tóth, I. V.; Rangel, A. O. S. S.; Anal. Methods 2013, 5, 585.
72. Cohen, N.; Sabhachandani, P.; Sarkar, S.; Kahanovitz, L.; Lautsch, N.; Russell, S. J.; Konry, T.; Microchim. Acta 2017, 184,835 .

73. Chen, X. W.; Zou, A. M.; Chen, M. L.; Wang, J. H.; Dasgupta, P. K.; Anal. Chem. 2009, 81, 1291.

74. Dominguez, R. B.; Alonso, G. A.; Munoz, R.; Hayat, A.; Marty, J. L.; Sens. Actuators, B 2015, 208, 491.

75. Satínský, D.; Solich, P.; Chocholous, P.; Karlicek, R.; Anal. Chim. Acta 2003, 499, 205.

76. Batista, A. D.; Chocholous, P.; Satínský, D.; Solich, P.; Rocha, F. R. P.; Talanta 2015, 133, 142.

77. Batista, A. D.; Rocha, F. R. P.; Anal. Methods 2015, 7, 4371.

78. Maya, F.; Horstkotte, B.; Estela, J. M.; Cerda, V.; TrAC, Trends Anal. Chem. 2014, 59, 1.

79. Nascimento, C. F.; Brasil, M. A. S.; Costa, S. P. F.; Pinto, P. C. A. G.; Saraiva, M. L. M. F. S.; Rocha, F. R. P.; Talanta 2015, $144,1189$.

80. Frizzarin, R. M.; Portugal, L. A.; Estela, J. M.; Rocha, F. R. P.; Cerdà, V.; Talanta 2016, 148, 694.

81. Maratta, A.; Martinez, L. D.; Pacheco P.; Microchem. J. 2016, 127, 199.

82. Strzelak, K.; Koncki, R.; Analyst 2015, 140, 7271.

83. Kubán, P.; Karlberg, B.; Anal. Chim. Acta 2009, 648, 129.

84. Duffy, G.; Regan, F.; Analyst 2017, 142, 4355.

85. Jiménez-López, J.; Santos, J. L. M.; Ortega-Barrales, P.; Ruiz-Medina, A.; Food Anal. Methods 2017, 10, 2412.

Submitted: October 14, 2017 Published online: February 6, 2018 